EXTENDED REPORT

\title{
Use of preoperative assessment of positionally induced cyclotorsion: a video-oculographic study
}

\author{
R Becker, T H Krzizok, H Wassill
}

Br J Ophthalmol 2004;88:417-421. doi: 10.1136/bjo.2003.025783

\begin{abstract}
See end of article for authors' affiliations

.....................

Correspondence to: Dr Ralph Becker, Department of Strabismology and Neuroophthalmology, University of Giessen Friedrichstrasse 18, D-35385 Giessen, Germany; ralph-becker@ web.de

Accepted for publication 11 July 2003
\end{abstract}

Purpose: Positionally induced cyclotorsion could be an important factor concerning correction of astigmatism in refractive surgery. The method of binocular three dimensional infrared video-oculography (3D-VOG) was used to determine a possible influence of body position on cyclotorsion.

Methods: 38 eyes (19 healthy subjects, median value of age 25.5) with normal binocular vision were examined using 3D-VOG. This method records ocular motions and positions of both eyes simultaneously in the $x, y$, and $z$ axis. Cycloposition of the eyes was recorded first in a seated position (both eyes open, test 1), then in a supine position (right eye closed, test 2), occlusion of both eyes (test 3), both eyes open (test 4). Cyclovergence was calculated as the difference between the right and the left eye positions.

Results: The range of cyclotorsion of the right and left eye in all four tests was between $1.13^{\circ}$ excyclotorsion and $0.34^{\circ}$ incyclotorsion. There was no statistically significant difference of the median values for torsion for the four test situations. Concerning the influence of body position on cyclotorsion, a statistically significant difference between the different test positions and settings did not exist. Median values for right/left torsion/cyclovergence were: $0.17 / 0.04 / 0.02$ (test 1), $-0.31 /-0.71 /-0.16$ (test 2), -1.09 / $-0.60 / 0.82$ (test 3), 0.28/0.28/-0.82 (test 4).

Conclusions: Cyclotorsion does not significantly change between seated and supine position in subjects with normal binocular vision and stable fixation. In these subjects, an erroneous refractive surgery due to incorrect measurement of the axis of astigmatism in the seated position and performing the refractive surgery in the supine position, is very unlikely.
P sitionally induced in- or excyclotorsion could be an important factor concerning correction of astigmatism in refractive surgery. Before performing refractive surgery, the axis of astigmatism is usually measured in a seated position, while the procedure of correction itself (for example, LASIK, LASEK, and PRK) takes place in a supine position.

Recently, the influence of binocular versus monocular fixation while measuring the axis of astigmatism was described by Tjon-Fo-Sang et al using a hand held keratometer. They found, that under monocular viewing conditions, significant cyclotorsion with a possible risk of undercorrection of myopic astigmatism may occur. Concerning body position itself, an influence on ocular torsion could not be found, similar to the results of Smith and Talamo, ${ }^{2}$ who used Maddox double rod measurements. Because of the relevance of positionally induced cyclotorsion for refractive surgery, it is worthwhile examining this with a new measurement device. In this study, we used the method of binocular three dimensional infrared video-oculography to determine the possible influence of body position on cyclotorsion. The method was developed to search for the causes of space sickness as early as $1980 .^{3}$ This non-invasive method can record ocular motions and positions of both eyes simultaneously in all degrees of freedom thus allowing a 3D positional analysis with unrestricted gaze and unrestricted head movements. Recently, Schworm et al used the same method for measurement of ocular counterrolling. ${ }^{4}$ This well known physiological reaction to the influence of gravity and acceleration in the vestibular system might have another consequence than the aim of this study-that is, a subject in the supine position should be positioned in a straight head position without head tilt. Gordes et al ${ }^{5}$ reported relatively large changes of ocular torsion depending on body position, also using the method of binocular 3D infrared video- oculography. Another recent study by Swami et al ${ }^{6}$ also described an influence of body position on cyclotorsion. Before excimer laser vision correction surgery, they marked each eye while the patient was seated upright. Before beginning the laser procedure, rotational malposition was measured on the supine patient and a torsional misalignment with a mean of 4.1 degrees was found.

\section{METHODS}

\section{Subjects}

Thirty eight eyes from 19 subjects with no known abnormalities of their visual system and stereopsis of at least 40 seconds of arc were included in the study. Median value of age was 25.5 years. The subjects had a refractive error of 0.08 $\mathrm{D}$ (median value) of the mean spherical equivalent and standard deviation of 0.08. All subjects were positioned comfortably, first in a seated upright and then in a supine position. Strabismus (especially dissociated vertical divergence, DVD) and any organic eye disease were excluded by thorough ophthalmological examination including biomicroscopy, indirect ophthalmoscopy, refractometry or retinoscopy in cycloplegia, cover test, assessment of ocular motility, prism and cover test, verification of stable, central fixation by using the so called "Haidinger Büschel" and afterimage, determination of stereopsis by the Lang stereotest and the Titmus test. All subjects had normal visual acuity in both eyes and random dot stereopsis of at least 40 seconds of arc. The study followed the Declaration of Helsinki. The nature of the study had been clearly explained and informed consent was obtained from all subjects before testing.

Abbreviations: 3D-VOG, three dimensional video-oculography; DVD, dissociated vertical divergence; LT, left torsion; RT, right torsion 


\section{Binocular infrared three dimensional video- oculography}

For recording eye positions, the method of three dimensional infrared video-oculography (3D-VOG) was used. The patient had to put on a mask, which is similar to a diving mask, as shown in figure 1 . In order to obtain a stable position, a chin rest together with a bite bar was used.

The device is formed of two high resolution video cameras and 3 infrared LED sources in a pair of goggles and a computer with especially designed video cards. The system is commercially available (Senso Motoric Instruments $\mathrm{GmbH}$, Teltow, Germany). The position of the eyes is analysed in three dimensions. This is performed for the calculation of horizontal and vertical position movements and deviations by the "black pupil technique" - that is, by infrared measurement and localisation of the individually calibrated pupil. The difference in horizontal and vertical position between both eyes gives the angle of deviation, and the graph of these positions, measured all 0.02 seconds, leads to the horizontal and vertical eye movements. We will use the term cycloposition for expression cyclotorsion. Both describe the actual static deviation of the sole right or sole left eye in the sagittal or torsional axis from the null position. The term cycloduction describes a dynamic deviation or movement in this axis. Thus, connecting the large amount of data for cycloposition will give a graph showing the cycloduction or cycloductional movement. It is important to recognise that all measurements for cycloposition reflect a relative measurement, which starts from 0 at an arbitrary point (time zero seconds) and position $\left(0^{\circ}\right.$ cycloposition). The cyclovergence was calculated as the difference between the right and the left eye positions in the torsional axis-that is, the $y$ axis of Fick's coordinate system (RE-LE).

The determination of the cycloposition and cycloduction of each eye (the y axis in Fick's coordinate system), ${ }^{7}$ is enabled by measuring a selected iris segment and its deviations (fig 2). Iris structures are overlayed and compared in order to determine the amount of rotation around the visual axis. Illumination of each eye was provided by three infrared LED sources with a wavelength of $920 \mathrm{~nm}$ (intensity $<1 \mathrm{~mW}$ / $\mathrm{cm}^{2}$ ). Torsional movements were sampled with a frequency of $50 \mathrm{~Hz}$. A measurement was taken every 0.02 seconds. Thus, 3000 measurements for both torsion of the right and left eye (cycloposition) could be obtained during a measurement of 1 minute. In studies comparing the accuracy of binocular infrared video-oculography with other techniques, a more detailed description of the method can be found. ${ }^{8}{ }^{9}$

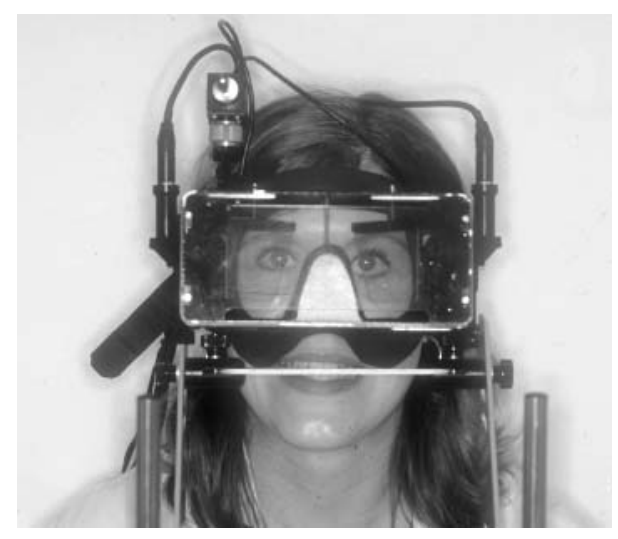

Figure 1 Front view of the recording mask on a subject in seated position with both eyes open. In order to obtain a stable position, a chin rest and bite bar were used.

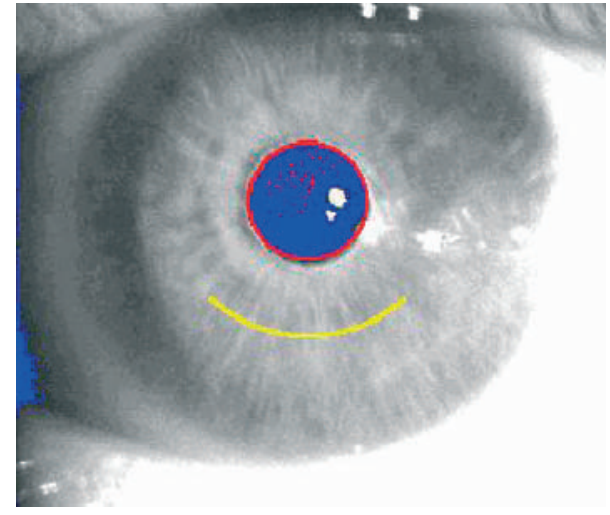

Figure 2 Yellow marked iris segment serving as a reference profile for assessment of ocular cyclotorsion by subsequent correlation of the reference profile with that of neighbouring segments for each video frame.

Positive values express incyclotorsion and negative values express excyclotorsion of the eye.

Cycloposition of the eyes was recorded first in a seated position with both eyes open (test 1) then, in order to obtain a quite realistic experimental setting, in a supine position, beginning with the right eye closed (test 2), then with occlusion of both eyes (test 3), followed by a fourth measurement with both eyes open in the supine position (test 4). Occlusion of one or both eyes was achieved with the help of a piece of metal put on the front side of the recording mask serving as an occluder. Each of these four tests was recorded for 1 minute allowing measurement of 3000 positions. A diagonal cross of $3^{\circ}$ presented in primary gaze served as a fixation target. Primary gaze is determined clinically and not derived from experimental calculations of Listing's plane. ${ }^{10}{ }^{11}$ The fixation distance for the seated and supine position was $4 \mathrm{~m}$. The calibration made at the initial position at the starting time (zero seconds) should give the positional values $0 ; 0 ; 0$. According to the aim of our study and the negligible horizontal and vertical deviations, the key measurement was that of the cycloposition.

Data collection was performed with the software that accompanies the system. The ocular cyclotorsion was assessed by calculation of the angular displacement of the position of a defined iris segment (fig 2). Therefore, grey levels of a specific iris segment serving as a reference profile were extracted and a correlation of the profile with the neighbouring segments was performed for each video frame. The software that accompanies the system allows the calculation of the so called quality factor Q (between 0.0 and 1.0) representing the concordance between the reference profile and that of the same iris segment of each consecutive frame during recording. The instruction manual suggests a quality factor of 0.3 or better in order to reduce the risk of false data. In our examinations, the quality factor was higher than 0.8 .

\section{Statistical analyses}

The statistical calculations for comparison of ocular cyclotorsion of both eyes were performed using the Wilcoxon matched pairs signed rank test. In order to work out the relationship between body position and induced in- or excyclotorsion concerning the different test situations (test $1-4)$, we used a multivariate analysis of variance $(\alpha=0.05)$. Median values and $95 \%$ confidence intervals for right torsion (RT), left torsion (LT), and cyclovergence for the four tests were calculated. 


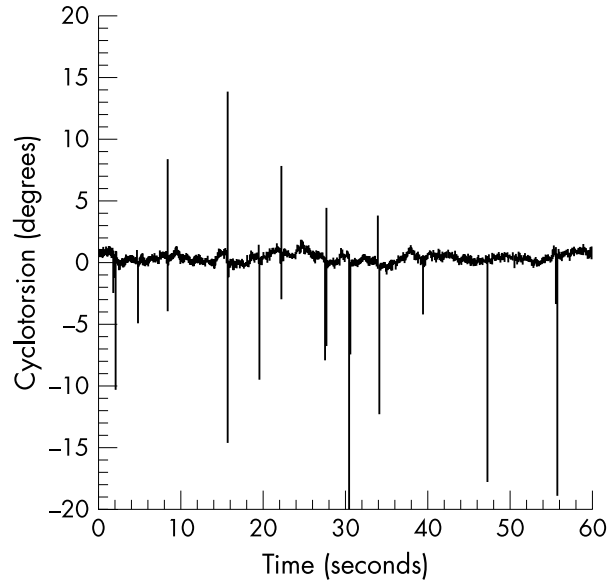

Figure 3 Cyclotorsion of the right eye for one subject with both eyes open in a seated position (test 1). The recording time was 1 minute and data are expressed in degrees with positive values expressing incyclotorsion and negative values expressing excyclotorsion of the eye. The vertical peaks are caused by blinking.

\section{RESULTS}

The measured cyclotorsional deviations were actually very small. In the seated (test 1 ) as well as in the supine position with both eyes opened (tests 1 and 4 ), the cycloposition of both eyes was located around $0.11^{\circ}$ of incycloposition. This "rest position" shifts in the supine position with occlusion of either one (test 2) or both eyes (test 3) to a cycloposition penduling around $1^{\circ}-2^{\circ}$ of excycloposition. These oscillations of both eyes were not conjugated-that is, they were in some of the small time intervals clockwise, in other time intervals anti-clockwise. The shift was detectable in the cycloposition of the right and left eye equally in all patients. In the supine position with opening of both eyes, cyclotorsion showed fewer artifacts compared with the supine position with both eyes closed, but was still differing more from zero than in the seated position, although statistically not significantly. In a supine position with occlusion of one eye, cyclotorsion of both eyes was higher, but not statistically significant, than in the seated position. When both eyes were closed in the supine position, cyclotorsion was less regular with a bigger difference from zero compared with the seated and supine position with occlusion of only one eye (see table 1).

Statistical analysis of the different test positions did not show any positionally induced cyclotorsion-that is, we did not find a statistically significant difference of the eyes' cyclotorsion between seated and supine position either with or without occlusion of one or both eyes. There also were no statistically significant differences between right and left eyes for the seated and supine test positions.

Figures 3 to 8 show examples of the cyclotorsion of the right/left eye for one of the normal subjects for the four test conditions. All subjects showed similar changes of in- and

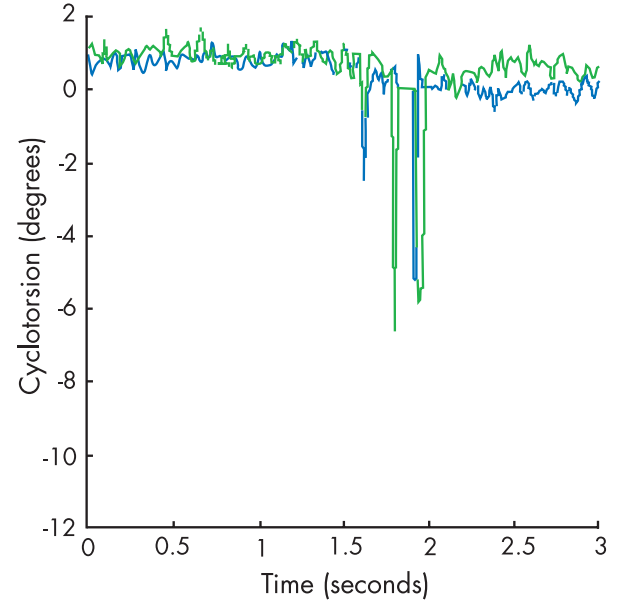

Figure 4 First 3 seconds of the cyclotorsion of both eyes (blue = right eye, green $=$ left eye) for the subject in figure 3 (test 1). The range of cycloposition of the right (left) eye is between $1.2^{\circ}\left(1.8^{\circ}\right)$ and $0.6^{\circ}\left(2.0^{\circ}\right)$ excyclotorsion.

excyclotorsion for the different test positions and settings. The vertical peaks-for example, in figure 4 peaks exceeding 1 degree of excyclotorsion-are artifacts caused by blinking. This could be proved by monitoring and observation of the patient's lids and eyes during the measurement. Figure 3 shows the cyclotorsion of the right eye for one subject with both eyes open in a seated position (test 1). Positive values express incyclotorsion and negative values express excyclotorsion of the eye. Figure 4 shows the first 3 seconds of the cyclotorsion of both eyes (blue line: right eye; green line: left eye) for the same subject referred to in figure 3 for test 1 . The range of cycloposition of the right (left) eye is between $1.2^{\circ}$ $\left(1.8^{\circ}\right)$ incyclotorsion and $0.6^{\circ}\left(2^{\circ}\right)$ excyclotorsion. When both eyes were open in the supine position (test 4 ), we found an oscillation of the eye position between an excycloposition of $1.4^{\circ}$ and and incycloposition of $2.4^{\circ}$ (fig 5). Figure 6 again shows the first 3 seconds for this test situation. While the range of cyclotorsion for the right eye was higher than for the left eye, a statistically significant difference did not exist. Figure 7 expresses cyclotorsion of the right eye for the same subject referred to in figures 3-6 with the right eye closed in a supine position (test 2 ) and in figure 8 , cyclotorsion of the right eye for the same subject with occlusion of both eyes in the supine position (test 3 ), achieved with the help of a piece of metal put on the front side of the recording mask, is shown. In this test situation, cyclotorsion is with a bigger difference from zero compared with the seated and supine position with occlusion of only one eye.

In all examinations, a quality factor higher than 0.8 could be achieved representing data with a high quality level with a minimum of false positive or false negative values. Examinations took 4 minutes in total for each subject. For

Table 1 Median values (95\% confidence interval) for 38 eyes from 19 subjects for right torsion (RT), left torsion (LT), and cyclovergence for the four tests (degree) for a measurement of one minute.

\begin{tabular}{lllll}
\hline Measurement & Test 1 & Test 2 & Test 3 & Test 4 \\
\hline RT & $0.17(-0.06$ to 0.4$)$ & $-0.31(-2.42$ to -1.8$)-1.09(-2.47$ to 0.29$)$ & $0.28(-0.73$ to 1.29$)$ \\
LT & $0.04(-0.3$ to 0.38$)$ & $-0.71(-1.96$ to 0.54$)$ & $-0.60(-3.3$ to 2.1$)$ & $0.28(-1.28$ to 1.84$)$ \\
Cyclovergence & $0.02(-0.41$ to 0.45$)$ & $-0.16(-1.2$ to 0.88$)$ & $0.82(-0.54$ to 2.18$)$ & $-0.82(-3.59$ to 1.95$)$
\end{tabular}

Test 1: seated position, both eyes open; test 2: supine position, right eye closed; test 3: supine position, both eyes closed; test 4: supine position, both eyes open. Incyclotorsion is characterised by positive values and excyclotorsion by negative values. 


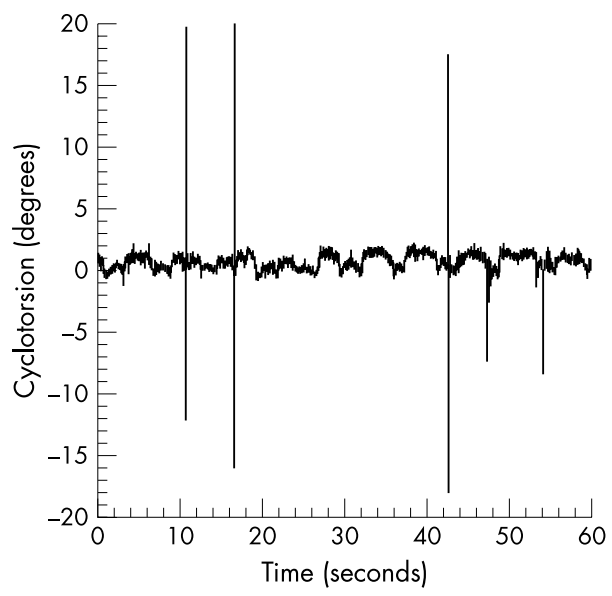

Figure 5 Cyclotorsion of the right eye for one subject (figs 3 and 4) with both eyes open in a supine position (test 4 ). The eye position oscillates between an excycloposition of $1.4^{\circ}$ and an incycloposition of $2.4^{\circ}$.

each of the four tests in one subject, 3000 measurements of ocular cyclotorsion were performed for each eye.

\section{DISCUSSION}

Positionally induced in- or excyclotorsion could be an important factor in the outcome of refractive surgery. Two possible mistakes with clinical significance may occur. Firstly, a simple difference of the axis of astigmatism between the sitting and supine position will lead not only to an erroneous correction of the astigmatism itself, but the whole spherocylindrical correction could be false. This applies especially to the modern flying spot excimer lasers. ${ }^{12}$ Secondly, an unpredictable cyclotorsional movement-that is, an oscillation of the y axis of each eye, will result in complex errors of the excimer laser ablation. This would make a tracking system for the torsional movement of the eye absolutely mandatory.

We used the method of binocular three dimensional infrared video-oculography in order to determine a possible influence of body position on cyclotorsion with a very accurate method, and we did not find any statistically significant positionally induced cyclotorsion. Our finding that body position does not have any influence on ocular torsion, is in accordance with previous studies by Tjon-FoSang ${ }^{1}$ and Smith, ${ }^{2}$ but as they used different methods, a direct comparison with their results is not possible. Tjon-FoSang ${ }^{1}$ found in $13 \%$ of a total of 15 persons with normal bonocular vision, that statistically significant excyclotorsion occurred when changing from binocular to monocular fixation in a seated position. The same occurred in the supine position in the study of Tjon-Fo-Sang, ${ }^{1}$ in which three subjects $(20 \%)$ showed excyclotorsion when the fixation changed. Therefore Tjon-Fo-Sang et al found only an influence of the way of fixation-that is, if the patient has binocular fixation with stereopsis, or if the patient has only monocular fixation.

We found that in the seated and supine position with both eyes open, the cycloposition of the right eye and the left eye, respectively, was located approximately around $1^{\circ}$ of incycloposition. This "rest position" shifts in the supine position with either one or both eyes closed to a cycloposition penduling around $1^{\circ}$ to $2^{\circ}$ of excycloposition. This shift was detectable in the cycloposition of the right and left eye in most patients. In contrast to Gordes et al, all measurements revealed very small deviations from zero in the y axis of the eye in Fick's coordinate system. As Gordes et al used the same measurement device, one explanation for their persis-

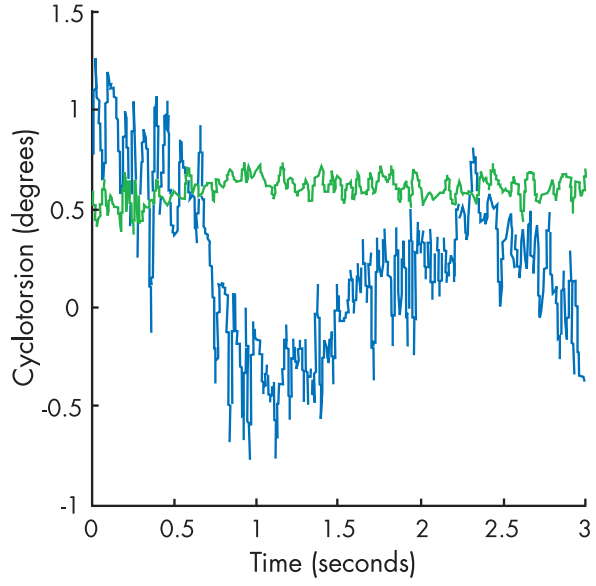

Figure 6 First 3 seconds of the cyclotorsion of both eyes (blue $=$ right eye, green $=$ left eye) for the subject referred to in figures $3-5$ for test 4 (that is, both eyes open in a supine position). As in other figures, positive values express incyclotorsion and negative express excyclotorsion of the eye. The range of cyclotorsion of the right eye $\left(1.28^{\circ}\right.$ to $\left.-0.78^{\circ}\right)$ of this subject is higher than of the left eye $\left(0.75^{\circ}\right.$ to $\left.-0.37^{\circ}\right)$ for this time interval, but the difference in ranges is not statistically significant.

tent higher values for cyclotorsion could be that the quality factor was high in our measurements $(>0.8)$. It is well known that even a small inaccuracy during the VOG measurement may lead to higher values of cycloposition from zero. Another explanation might be that humans without normal binocular vision and stereopsis will have higher deviations of the cycloposition from zero. Liesch and Simonsz showed that the disturbance of binocular vision for some days will cause sursoadduction and excyclotropia of the occluded eye. ${ }^{13}$ As a consequence, it should be pointed out that subjects with subnormal binocular vision or with unstable fixation in relation to reduced visual acuity or non-central fixation will have a much higher cyclotorsion in a significant range. We plan to perform another experiment on people with reduced or without binocular vision caused by strabismus, microtropia, anisometropia, or other reasons as well as in patients with unstable fixation. It will also be interesting to see if people with dissociated vertical deviation (DVD), which were not included in our study, will show any different behaviour regarding cyclotorsion for the different test situations. We know that DVD can present with excycloduction of the elevated eye and incyloduction of the

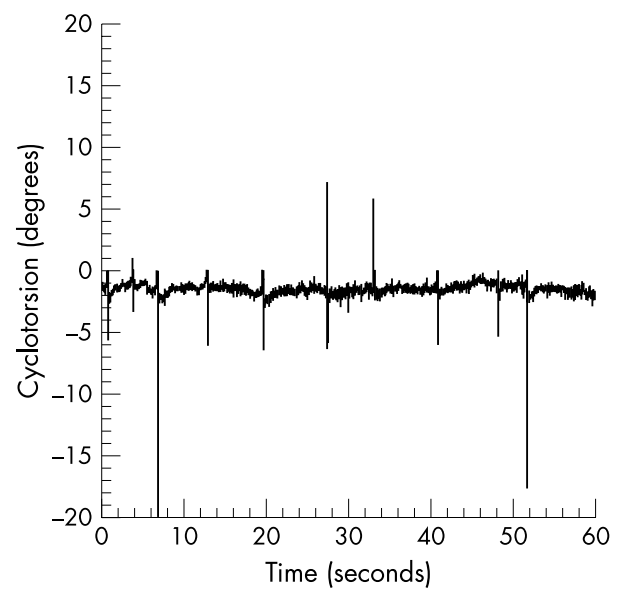

Figure 7 Cyclotorsion for the right eye of the subject referred to in figures 3-6 with the right eye closed in a supine position (test 2). 


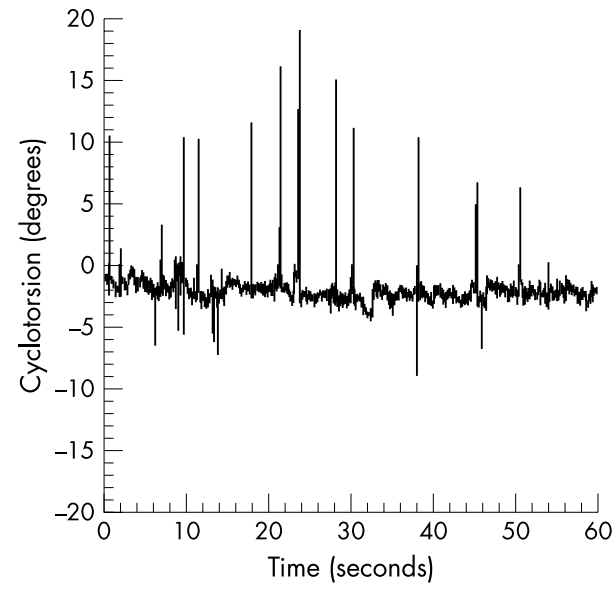

Figure 8 Cyclotorsion for the right eye for one subject (figs 3-7) with both eyes closed in a supine position (test 3 ). Cyclotorsion is with a bigger difference from zero compared with the seated and supine position with closure of one eye.

fixating eye. Enright" ${ }^{14}$ created the word "cyclotorsional noise" as the measured cyclotorsional deviations were very small.

Our experimental setting is quite realistic for a LASIK or LASEK situation, which are currently the most common refractive surgeries. If the patient has normal binocular vision and stable fixation of both eyes, it will in the future neither be necessary to measure corneal topography or ocular aberrations in the supine position in order to avoid uneventful torsional deviations, nor will it be mandatory to implement a device like this 3D-VOG as a tracking device to control torsional deviations. Further investigations will show if our results also apply to subjects without normal binocular vision or unstable fixation. If these subjects show higher cyclotorsions or fluctuations between the sitting and supine position, tracking devices to control the torsion of the eyes will be useful, as described by MacRae et al. ${ }^{12}$

\section{ACKNOWLEDGEMENTS}

The authors would like to thank Dr Michael Armbrust, MD, Eye Clinic Lohr, Partensteiner Str 6, 97816 Lohr for advice and useful discussions.

\section{Authors' affiliations}

R Becker, T H Krzizok, H Wassill, Department of Strabismology and

Neuroophthalmology, University of Giessen, Germany

\section{REFERENCES}

1 Tion-Fo-Sang M, de Faber J-T, Kingma C, et al. Cyclotorsion: A possible cause of residual astigmatism in refractive surgery. $J$ Cataract Refract Surg 2002;28:599-602

2 Smith EM Jr, Talamo JH. Cyclotorsion in the seated and supine patient. $J$ Cataract Refract Surg 1995;21:402-3.

3 Clarke HC, Teiwes W, Scherer H. Video-oculography-an alternative method for measurement of three-dimensional eye movements. In: Schmid R, Zambarbieri D, eds. Oculomotor Control and Cognitive Processes. Amsterdam: Elsevier Science Publishers BV, 1980:431-43.

4 Schworm H, Ygge J, Pansell T, et al. Assessment of ocular counterroll during head tilt using binocular video oculography. Invest Ophthalmol Vis Sci 2002;43:662-7.

5 Gordes RS, Vaseghi S, Pansell T, et al. Influence of the body position to cyclorotation in healthy probands. Ophthalmologe 2002;99(suppl 1): 142.

6 Swami AU, Steinert RF, Osborne WE, et al. Rotational malposition during laser in situ keratomileusis. Am J Ophthalmol 2002;133:561-2.

7 Fick A. Neue Versuche über die Augenstellungen. In: Moleschott J, ed. Untersuchungen zur Naturlehre des Menschen und der Thiere. (V). Frankfurt: Meidinger, 1858:193-233.

8 Scherer $\mathrm{H}$, Teiwes W, Clarke AH. Measuring three dimensions of eye movement in dynamic situations by means of videooculography. Acta Otolaryngol 1991;111:182-7.

9 Teiwes W, Merfeld DM, Young LR, et al. Comparison of the scleral search coil and video-oculography techniques for three-dimensional eye-movement measurement. In: Fetter M, Haslwanter T, Misslich H, Tweed D, eds. ThreeDimensional Kinematics of Eye, Head and Limb Movements. Amsterdam: Overseas Publishers Association, 1997:429-43.

10 Nakayama K. A new method of determining the primary postion of the eye using Listing's Law. Am J Optom Physiol Opt 1978;55:331-6.

11 Ferman L, Collewijn H, van den Berg AV. A direct test of Listing's law-I. Human ocular torsion measured in static tertiary positions. Vision Res 1987;27:929-38.

12 MacRae SM, Krueger RR, Applegate RA. Customized corneal ablation: the quest for supervision. SLACK Incorporated 2001:158.

13 Liesch A, Simonsz HJ. Up- and downshoot in adduction after monocular patching in normal volunteers. Strabismus, 1 1993:25-29.

14 Enright JT. Stereopsis, cyclotorsional "noise" and the apparent vertical. Vision Res 1990;30: 1487-97. 